Department of Applied Mathematics

Faculty of EEMCS

(5)

University of Twente

The Netherlands
P.O. Box 217

7500 AE Enschede

The Netherlands

Phone: +31-53-4893400

Fax: $+31-53-4893114$

Email: memo@math.utwente.nl

www.math.utwente. $\mathrm{nl} /$ publications

Memorandum No. 1744

Spaces of boundary values

related to a multipoint

version of the KP-Hierarchy

G.F. HeLmincK

December, 2004

ISSN 0169-2690 


\title{
Spaces of boundary values related to a multipoint version of the $K P$-hierarchy
}

\author{
G.F. Helminck \\ Faculty of Mathematical Sciences \\ University of Twente \\ P.O. Box 217, 7500 AE Enschede \\ The Netherlands \\ e-mail: helminckgf@cs.utwente.nl
}

17 December 2004

\begin{abstract}
In this paper one considers a finite number of points in the complex plane and various spaces of boundary values on circles surrounding these points. To this geometric configuration one associates a Grassmann manifolds that is shown to yield solutions of a multipoint version of the linearization of the $K P$-hierarchy. These Grassmann manifolds are built in such a way that the determinant line bundle and its dual over them still make sense. The same holds for the so-called $\tau$-functions, determinants of certain Fredholm operators that measure the failure of equivariance at lifting the commuting flows of ths hierarchy to these bundles. Solutions of the linearization are described by wave functions of a certain type. They are perturbations of the trivial solution with the leading term of the perturbation determining the type. One concludes with showing that, if a plane in the Grassmann manifold yields wave functions of different types, they are connected by a differential operator in the coordinates of the flows.
\end{abstract}

Subject classification: 22E65, 22E70, 35Q58, 58B25.

Keywords: multipoint KP-hierarchy, boundary values, Grassmann manifold.

\section{Introduction}

As Sato has indicated, see e.g. [SS83], spaces of boundary values on the circle yield solutions of the $K P$-hierarchy, which are the compatibility conditions for an infinite linear system, called its linearization, see e.g. [DJKM83].

For the case of the square integrable functions on the unit circle this connection was treated extensively in [SW85]. There they considered a Grassman manifold consisting of planes close to that of the square integrable functions on the unit circle that have an analytic continuation to infinity. The flows lying at the basis of the linearization correspond to the group of holomorphic functions on the unit disc around infinity and vanishing there. To each subspace in this manifold they associated a solution of this linearization, which carries the name wavefunction or Baker-Akhiezer function. These wavefunctions can be expressed in so-called $\tau$-functions, Fredholm-determinants, which have an interpretation in terms of the determinant bundle over this Grassman manifold and its dual. A Lie algebraic interpretation of these functions was given by V.Kac, see e.g. [Kac88].

In [Dic94] it was suggested to consider a multipoint version of the $K P$-hierarchy corresponding to $n$ points $\left\{a_{1}, \ldots, a_{n}\right\}$ in $\mathbb{C}$ and the point infinity. In this paper the multipoint analogue of the linearization of the $K P$-hierarchy is taken under consideration. The group of flows that are a proper 
translation of the ones for the $K P$-hierarchy consists of the functions that are holomorphic on the complement of circles around each of the points $a_{i}$ and that vanish at infinity. These continuous flows can be complemented with a natural lattice group that commutes with them. The next step will be to choose a number of disjoint circles around the points $\left\{a_{1}, \ldots, a_{n}\right\}$ and on each circle an appropriate space of boundary values. The total space of boundary values possesses a decomposition to which one associates a suitable Grassmanian. For each plane in this manifold one can construct solutions of the linearization of a specific nature depending of the lattice points. Solutions corresponding to the same plane and different lattice points are shown to be related by a differential operator.

A specification of the content of the various sections is as follows: the first is devoted to an algebraic set-up for the linear evolution equations corresponding to the flows. In the next section the properties of the spaces of boundary values are discussed and the basic decomposition is presented. It is different of the one suggested in [Dic94]. The subsequent section starts with a description of the Grassmanian, it discusses furthermore its structure, various realizations and how the flows fit in. Note that already in the square integrable case, as considered e.g. in [SW85], the Grassmanian introduced here is significantly more general. Despite this general character one can still define the determinant bundle and its dual over this manifold and that is done in the following section. The final section contains the construction for each plane in the connected component of the Grassman manifold of multipoint wavefunctions of a certain type and a discussion of the dependence of the lattice.

\section{An algebraic set-up for the linearization}

The independent variables $\left\{t_{i} \mid i \geq 1\right\}$ occurring in the $K P$-hierarchy are the coordinates of the commuting flows of this integrable system,

$$
\gamma(t, z)=\exp \left(\sum_{i=1}^{\infty} t_{i} z^{-i}\right)
$$

where $t$ is a short-hand notation for the $\left\{t_{i}\right\}$. The nonlinear equations of the $K P$-hierarchy are obtained by cross-differentiation of the so-called linearization. In this system the relevant differential operators of the $K P$-hierarchy act on objects like

$$
\left\{\sum_{i \geq N} a_{i}(t) z^{i}\right\} \gamma(t, z)
$$

which are formal products of a power series in $z$, depending of $t$, that has at the utmost a pole in $z=0$ and an exponential factor corresponding to the commuting flows that describes the essential singularity around zero. Often the role of the points zero and infinity is interchanged, but for the present generalization one sticks to the decomposition (2). Consider thereto, instead of the point zero, $n$ different points $a_{1}, \ldots, a_{n}$ in $\mathbb{P}^{1}(\mathbb{C}) \backslash\{\infty\}$. The exponential factor describing the essential singularity around each of the points $a_{1}, \ldots, a_{n}$ has the form

$$
\gamma(t, z):=\exp \sum_{k=1}^{n} \sum_{l=1}^{\infty} t_{l}^{(k)}\left(z-a_{k}\right)^{-l}
$$

Here $t$ is again the short-hand notation for the collection of coordinates $\left\{t_{l}^{(k)}\right\}$. It is convenient to use a notation related to this group of transformations. One will write

$$
\xi_{k}(z, t)=\sum_{l=1}^{\infty} t_{l}^{(k)}\left(z-a_{k}\right)^{-l} \quad \text { and } \quad \xi(z, t)=\sum_{k=1}^{n} \xi_{k}(z, t) .
$$


Let $\mathcal{R}$ be a complex algebra of functions in the $\left\{t_{l}^{(k)}\right\}$ that is stable under all the partial derivatives $\partial_{k}^{(i)}:=\frac{\partial}{\partial t_{k}^{(i)}}$. For each $k, 1 \leq k \leq n$, let $\mathscr{H}_{k}(\mathcal{R})$ be the space of all power series in $z-a_{k}$ with coefficients from $\mathcal{R}$ that only have a pole in $z=a_{k}$, i.e.

$$
\mathscr{H}_{k}(\mathcal{R})=\left\{\sum_{s \geq N} a_{s}\left(z-a_{k}\right)^{s} \mid a_{s} \in \mathcal{R}, \quad N \in \mathbb{Z}\right\} .
$$

The analogue of the other factor in decomposition (2) is taken from the space

$$
\mathscr{H}(\mathcal{R}):=\oplus_{k=1}^{n} \mathscr{H}_{k}(\mathcal{R}) .
$$

The linearization in the multipoint setting is now realised on the space $\mathcal{M}(\mathcal{R})$ of formal products of elements of $\mathscr{H}(\mathcal{R})$ and the exponential factor $\gamma(t, z)$. Note that we have for each $s \in \mathbb{Z}$ a natural action of $\left(z-a_{k}\right)^{s}$ on the whole space $\mathscr{H}(\mathcal{R})$. On the $k$-th factor this action consists of multiplication with $\left(z-a_{k}\right)^{s}$ and for $i \neq k$ it acts on the $i$-th component by multiplying with the powerseries $((z-$ $\left.\left.a_{i}\right)+a_{i}-a_{k}\right)^{s}$. This enables you to define an $\mathcal{R}\left[\partial_{k}^{(i)}\right]$-module structure on $\mathcal{M}(\mathcal{R})$ by extending the following actions: for each element

$$
\psi=\left(\ldots, \sum_{l} h_{l}^{(k)}\left(z-a_{k}\right)^{l}, \ldots\right) \gamma(t, z)
$$

in the space $\mathcal{M}(\mathcal{R})$ and each $r \in \mathcal{R}$ one defines

$$
\begin{aligned}
r . \psi:= & \left(\ldots, \sum_{l} r h_{l}^{(k)}\left(z-a_{k}\right)^{l}, \ldots\right) \gamma(t, z) \\
\partial_{s}^{(i)}(\psi):= & \left(\ldots, \sum_{l} \partial_{s}^{(i)}\left(h_{l}^{(k)}\right)\left(z-a_{k}\right)^{l}, \ldots\right) \gamma(t, z) \\
& +\left(\ldots, \sum_{l} h_{l}^{(k)}\left(z-a_{k}\right)^{l}, \ldots\right)\left(z-a_{i}\right)^{-s} \gamma(t, z) .
\end{aligned}
$$

Now that one can consider differential equations w.r.t. the $\left\{\partial_{k}^{(i)}\right\}$ inside $\mathcal{M}(\mathcal{R})$, one still wants to fix a gauge of the eigenfunctions, since there is a natural group of transformations on $\mathcal{M}(\mathcal{R})$ that commutes with these derivatives. Recall that the group $\Sigma$ given by

$$
\Sigma=\left\{\sigma(\underline{m}):=\prod_{k=1}^{n}\left(z-a_{k}\right)^{m_{k}} \mid \underline{m}=\left\{m_{k}\right\} \in \mathbb{Z}^{n}\right\}
$$

acts on the space $\mathcal{H}(\mathcal{R})$ from the right and this action is extended to $\mathcal{M}(\mathcal{R})$ by

$$
h \gamma(t, z) \mapsto h \sigma(\underline{m}) \gamma(t, z) .
$$

An element $\psi \in \mathcal{M}(\mathcal{R})$ is called of type $(i, \sigma(\underline{m}))$ if it can be written as

$$
\psi=\hat{\psi} \sigma(\underline{m}) \gamma(t, z)
$$

, where $\hat{\psi} \in \mathcal{M}(\mathcal{R})$ has the form

$$
\hat{\psi}=\left(\ldots, \sum_{l \geq-1} h_{l}^{(k)}\left(z-a_{k}\right)^{l}, \ldots\right), \text { with } h_{-1}^{(i)}=1 \text { and } h_{-1}^{(k)}=0 \text { for } k \neq i .
$$

Now one can introduce the linearization of the multipoint $K P$-hierarchy. For an element $\psi_{i}^{(\sigma(\underline{m}))} \in$ $\mathcal{M}(\mathcal{R})$ of type $(i, \sigma(\underline{m}))$, it consists of all the equations such that

$$
\partial_{k}^{(j)}\left(\psi_{i}^{(\sigma(\underline{m}))}\right)=B_{k}^{(j, i)}\left(\psi_{i}^{(\sigma(\underline{m}))}\right), k \geq 1,1 \leq j \leq n,
$$


where $B_{k}^{(j, i)}$ is a differential operator of order $k$ in $\partial_{j}:=\partial_{1}^{(j)}$ with coefficients from $\mathcal{R}$. Following the terminology used in the case of the $K P$-hierarchy, one calls $\psi_{i}^{(\sigma(\underline{m}))}$ a wavefunction of the multipoint $K$ P-hierarchy of type $(i, \sigma(\underline{m}))$.

The equations of the multipoint $K P$-hierarchy are then the compatibility conditions for this linearization, which amount to nonlinear differential equations for the coefficients of $\hat{\psi}_{i}^{(\sigma(\underline{m}))}$ that can in a compact way be written as

$$
\left\{\partial_{m}^{(s)}\left(B_{k}^{(j, i)}\right)-\partial_{k}^{(j)}\left(B_{m}^{(s, i)}\right)+\left[B_{k}^{(j, i)}, B_{m}^{(s, i)}\right]\right\}\left(\psi_{i}^{(\sigma(\underline{m}))}\right)=0 .
$$

Remark 1 Note that if one considers a wavefunction $\psi$ of the multipoint K P-hierarchy of type $(i, \sigma(\underline{m}))$ merely for the variables $\left\{t_{l}^{(k)} \mid l \geq 1, k\right.$ fixed $\}$, then it is a solution of the KP-hierarchy. Hence the function $\psi$ is an intermingling of $n$ solutions of the KP-hierarchy.

To produce concrete solutions of the multipoint $K P$-hierarchy one constructs an analytic setting that generates elements of $\mathcal{M}(\mathcal{R})$ of type $(i, \sigma(m))$ for which the formal product is real and that satisfy all the equations (10). The next section intends to illustrate the freedom of choice one has in carrying out this construction.

\section{Spaces of boundary values}

Let the $\left\{D_{i} \mid 1 \leq i \leq n\right\}$ be disjoint open discs in $\mathbb{C}$ with center $a_{i}$, boundary $C_{i}$ and radius $r_{i}$. On each circle $C_{i}$ one assumes given a Banach space $H_{i}$ with norm $\|\cdot\|_{i}$. All these boundary values are joined together inside $H=\oplus_{i=1}^{n} H_{i}$, which is again a Banach space w.r.t. the norm

$$
\left\|\left(h_{i}\right)\right\|_{H}:=\sum_{i=1}^{n}\left\|h_{i}\right\|_{i}
$$

The spaces $H_{i}$ should satisfy a number of conditions that are listed here

Properties 3.1 (1) First of all, each space $H_{i}$ should consist of power series in $z-a_{i}$, i.e.

$$
H_{i}=\left\{f(z)=\sum_{n \in \mathbb{Z}} a_{n}\left(z-a_{i}\right)^{n}\right\}
$$

and all the $\left\{\left(z-a_{i}\right)^{n} \mid n \in \mathbb{Z}\right\}$ should belong to $H_{i}$.

(2) Inside $H_{i}$, one has the subspace

$$
H_{i}^{+}=\left\{f \in H_{i} \mid f(z)=\sum_{n \geq 0} a_{n}\left(z-a_{i}\right)^{n}\right\}
$$

of all series that possibly converge around $a_{i}$. It is the closure w.r.t. the norm $\|\cdot\|_{i}$ of the span of the $\left\{\left(z-a_{i}\right)^{n} \mid n \geq 0\right\}$. Moreover to each $f_{i} \in H_{i}^{+}$corresponds a function $F_{i}$ holomorphic on $D_{i}$ with boundary value $f_{i}$.

(3) The second subspace of $H_{i}$ is

$$
H_{i}^{-}=\left\{f \in H_{i} \mid f(z)=\sum_{n<0} a_{n}\left(z-a_{i}\right)^{n}\right\}
$$

the space of all series in $H_{i}$ that have a potential zero at infinity. Likewise, the space $H_{i}^{-}$is the closure w.r.t. the same norm of the space spanned by the $\left\{\left(z-a_{i}\right)^{n} \mid n<0\right\}$. For each 
$f_{i} \in H_{i}^{-}$corresponds a function $F_{i}$ holomorphic on the complement of $\overline{D_{i}}$ with boundary value $F_{i}(i):=f_{i}$. For all $k \neq i$ write $F_{i}(k)$ for the boundary value of $F_{i}$ at $C_{k}$, then each $F_{i}(k)$ should belong to $H_{k}^{+}$and the map $f_{i} \mapsto F_{i}(k)$ should be continuous.

(4) The spaces $H_{i}^{+}$and $H_{i}^{-}$generate $H_{i}: H_{i}=H_{i}^{+} \oplus H_{i}^{-}$and let for each $f_{i} \in H_{i}$ the corresponding decomposition be $f_{i}=f_{i}^{+}+f_{i}^{-}$. Moreover, the projections $p_{i}^{+}: H_{i} \mapsto H_{i}^{+}$and $p_{i}^{-}: H_{i} \mapsto H_{i}^{-}$are continuous.

(5) Finally, on each space $H_{i}$ multiplication with $z-a_{j}, 1 \leq j \leq n$, is a bounded invertible operator $M_{j}(i)^{-1}$. Consequently the same holds for multiplying with any of the $\left\{\left(z-a_{i}\right)^{n} \mid n \in \mathbb{Z}\right\}$.

Examples of such spaces are the $L^{p}$-functions on the boundary $C_{i}$ and variations on this theme. For the $K P$-hierarchy, Sato, see e.g. [SS83], realized that there is a great diversity in spaces of boundary values that lead to solutions of the $K P$-hierarchy. The analytic context for the $L^{2}$-boundary values was given in [SW85]. A class of Banach spaces yielding solutions to the $K P$-hierarchy occurs in [Dorf89].

Inside the space $H$ one considers the boundary values that are holomorphic inside all the circles, i.e. $H^{+}=\oplus_{i=1}^{n} H_{i}$, and those that are holomorphic outside all the discs $D_{i}$ and disappear at infinity,

$$
H^{*}=\overline{\operatorname{Span}\left(\left(\left(z-a_{i}\right)^{m}, \ldots,\left(z-a_{i}\right)^{m}\right) \mid 1 \leq i \leq n, m<0\right)} .
$$

By making use of the properties 3.1(2) and 3.1(3) and by invoking the edge of the wedge theorem as formulated e.g. in [dRoe78], one proves that the subspaces $H^{+}$and $H^{*}$ satisfy: $H^{+} \cap H^{*}=\{0\}$. From property 3.1(3) one sees that the map $p^{*}: H \mapsto H^{*}$ given by

$$
p^{*}\left(\left(f_{i}\right)\right)=\left(\sum_{j=1}^{n} F_{j}^{-}(1), \ldots, \sum_{j=1}^{n} F_{j}^{-}(n)\right),
$$

projects $H$ onto $H^{*}$ and that its restriction to $H^{-}$is a continuous bijection. Thus one arrives at

Proposition 1 The subspaces $H^{+}$and $H^{*}$ satisfy: $H=H^{*} \oplus H^{+}$.

This is the central decomposition leading to solutions of the linearization of the multipoint $K P$ hierarchy, since one can construct for any subspace of $H$ 'close' to $H^{*}$ a wavefunction of a certain type. The precise specification of what is meant by 'close" is done in the next section.

\section{The Grassmanian related to $H=H^{*} \oplus H^{+}$}

Before describing the infinite dimensional Grassman manifold, we recall some definitions and notations. For any closed subspace $W$ of $H$ let $i_{W}: W \mapsto H$ the natural continuous embedding. If $X$ and $Y$ are Banach spaces, then the space of Fredholm operators from $X$ to $Y$ consists of the continuous linear operators with a closed image and a finite dimensional kernel and cokernel. It is denoted by $\Phi(X, Y)$. The connected components of $\Phi(X, X)$ are described by the index

$$
i(A)=\operatorname{dim}(\operatorname{Ker}(A))-\operatorname{dim}(\operatorname{Coker}(A)) .
$$

Definition 1 The Grassman manifold $\operatorname{Gr}(H)$ corresponding to the decomposition $H=H^{*} \oplus H^{+}$ consists of all closed subspaces $W$ such that $p^{*} \circ i_{W}$ belongs to $\Phi\left(W, H^{*}\right)$.

This is a Banach manifold based on the space $L\left(H^{*}, H^{+}\right)$of bounded linear operators from $H^{*}$ to $H^{+}$and its connected components are given by

$$
\operatorname{Gr}_{k}(H):=\left\{W \in \operatorname{Gr}(H) \mid i\left(p^{*} \circ i_{W}\right)=k\right\}, k \in \mathbb{Z} .
$$


This manifold is a homogeneous space for various groups. The group that is presented here enables you to define the determinant bundle Det over $\operatorname{Gr}_{0}(H)$ and its dual Det* and needs the notion of nuclear operator. One recalls some of their features.

Let $E$ and $F$ be Banach spaces. Equip the topological dual $F^{*}$ with the operator norm. There is a natural embedding $v$ of $E \otimes F^{*}$ into the bounded operators $L(F, E)$, namely

$$
v\left(\sum_{j} e_{j} \otimes f_{j}^{*}\right)(x)=\sum_{j}<f_{j}^{*}, x>e_{j},
$$

where the $e_{j}$ belong to $E$, the $f_{j}^{*}$ to $F^{*}$ and $x$ to $F$. The image of $v$ are the finite dimensional operators in $L(F, E)$. On the tensor product $E \otimes F^{*}$ one defines

$$
\|u\|_{\mathfrak{n}}=\inf \sum_{j}\left\|e_{j}\right\|_{E}\left\|f_{j}^{*}\right\|_{F^{*}}
$$

where the infimum is taken over all $\sum_{j} e_{j} \otimes f_{j}^{*}$ equal to $u$. It is shown in [Grot55] that $\|\cdot\|_{\mathfrak{n}}$ defines a norm on $E \otimes F^{*}$, the Schatten norm. The completion of $E \otimes F^{*}$ w.r..t the Schatten norm is denoted by $E \hat{\otimes} F^{*}$. According to [Grot56], every element of $E \hat{\otimes} F^{*}$ can be written, though not uniquely, in the form

$$
\sum_{i=0}^{\infty} \lambda_{i}\left(e_{i} \otimes f_{i}^{*}\right), \text { with } \lambda_{i} \geq 0, \sum_{i=0}^{\infty} \lambda_{i}<\infty,\left\|e_{i}\right\|_{E} \leq 1,\left\|f_{i}^{*}\right\|_{F^{*}} \leq 1 .
$$

The map $v$ has a continuous extension $v: E \hat{\otimes} F^{*} \mapsto L(F, E)$ and the image of this last map is the space of nuclear operators $\mathcal{N}(F, E)$ from $F$ to $E$. Equiped with the quotient norm, also denoted by $\|\cdot\|_{\mathfrak{n}}$, it is a Banach space. In the case that $E$ and $F$ both equal a Hilbert space $K$, the nuclear operators are the trace class operators on $K$ and the norm $\|\cdot\|_{\mathfrak{n}}$ is the trace-norm.

Nuclear operators are stable w.r.t. composition from the left and right with bounded operators. More concretely, let $D$ be yet another Banach space and consider operators $X \in \mathcal{N}(F, E), Y \in$ $L(F, D)$ and $Z \in L(D, E)$. Then one has $Y \circ X \in \mathcal{N}(E, F)$ resp. $X \circ Z \in \mathcal{N}(D, F)$ and the following inequalities hold

$$
\|Y \circ X\|_{\mathfrak{n}} \leq\|Y\|\|X\|_{\mathfrak{n}} \text { and }\|X \circ Z\|_{\mathfrak{n}} \leq\|X\|_{\mathfrak{n}}\|Z\|
$$

Here $\|\cdot\|$ denotes again the operator norm. Using these properties of nuclear operators one shows that $G l_{\mathfrak{n}}(H)$, consisting of

$$
G l_{\mathfrak{n}}(H)=\left\{g=\left(\begin{array}{ll}
a & b \\
c & d
\end{array}\right) \in G l(H) \mid \begin{array}{cc}
a \in \Phi\left(H^{*}, H^{*}\right) & d \in \Phi\left(H^{+}, H^{+}\right) \\
c \in L\left(H^{*}, H^{+}\right) & b \in \mathcal{N}\left(H^{+}, H^{*}\right)
\end{array}\right\},
$$

is a group that acts transitively on $\operatorname{Gr}(H)$. In particular, the component $\mathrm{Gr}_{0}(H)$ is a homogeneous space for the subgroup

$$
G l_{\mathfrak{n}}^{(0)}(H)=\left\{g=\left(\begin{array}{ll}
a & b \\
c & d
\end{array}\right) \in G l_{\mathfrak{n}}(H) \mid i(a)=i(d)=0\right\},
$$

In $\mathrm{Gr}_{0}(H)$ we have the open dense subset of all planes $W$ such that $p^{*}: W \rightarrow H^{*}$ is a bijection. Like in the finite dimensional situation, this is called the big cell of $\operatorname{Gr}(H)$ and its elements are called tranverse to $H^{*}$.

For each $j, 1 \leq j \leq n$, consider the operator $M_{j}=\oplus_{i=1}^{n} M_{j}(i): H \mapsto H$ and let $M>0$ satisfy $M>\left\|M_{j}\right\|$, where $\|\cdot\|$ denotes the operator norm. For each such $M$ one considers the group of power series

$$
\tilde{\Gamma}(M)=\left\{\exp \sum_{k=1}^{n} \sum_{l=1}^{\infty} t_{l}^{(k)}\left(z-a_{k}\right)^{-l}\left|\sum_{k=1}^{n} \sum_{l=1}^{\infty}\right| t_{l}^{(k)} \mid M^{l}<\infty\right\}
$$


Its elements act continuously on $H$ by multiplication and if $\left(\begin{array}{cc}a & b \\ c & d\end{array}\right)$ is the decomposition of

$$
\sum_{k=1}^{n} \sum_{l=1}^{\infty} t_{l}^{(k)} M_{k}^{l}
$$

w.r.t. $H=H^{*} \oplus H^{+}$then a direct computation of the operator $b$ shows that it can be written in the form (17). Hence it is nuclear, so that the group of flows $\tilde{\Gamma}(M)$ embeds in this way into $G l_{\mathfrak{n}}^{(0)}(H)$. Likewise one can consider the action of the group $\Sigma$ on $H$ by multiplying on each factor with the element $\sigma(\underline{m})$ of $\Sigma$ and this gives you also an operator in $G l_{\mathfrak{n}}(H)$. The subgroup

$$
\Sigma^{(0)}=\left\{\sigma\left(\underline{m} \mid \sum_{k=1}^{m} m_{k}=0\right\}\right.
$$

corresponds to the elements that yield an operator in $G l_{\mathfrak{n}}^{(0)}(H)$. This last group is generated by the elements $\sigma_{i j}:=\frac{\left(z-a_{i}\right)}{\left(z-a_{j}\right)}$.

\section{The Line bundles Det and Det* over $\mathrm{Gr}_{0}(H)$}

Let $W$ belong to $\operatorname{Gr}_{0}(H)$. Since a Fredholm operator of index zero can be written as the sum of a finite dimensional one and an invertible operator, one can write $W$ as the image of an embedding $w: H^{*} \rightarrow H$ that decomposes w.r.t. $H=H^{*} \oplus H_{+}$as

$$
w=\left(\begin{array}{c}
w^{*} \\
w^{+}
\end{array}\right), \text {with } w^{*}-\text { Id finite dimensional and } w^{+} \text {bounded. }
$$

This class of embeddings is somewhat too restricted for the action of the group $G l_{\mathfrak{n}}^{(0)}(H)$. Henceforth one chooses to vary $w^{*}-$ Id inside a wider class of compact operators, the nuclear ones. The collection of all embeddings of this form is denoted by $\mathcal{P}^{*}$. The component $\mathrm{Gr}_{0}(H)$ can be described then as the quotient $\mathcal{P}^{*} / \mathcal{T}^{*}$, where $\mathcal{T}^{*}$ is the group defined by

$$
\mathcal{T}^{*}=\left\{t \in G l\left(H^{*}\right) \mid t-\mathrm{Id} \text { is nuclear }\right\},
$$

which acts on $\mathcal{P}^{*}$ by composition on the right. For operators from $H^{*}$ to $H^{*}$ of the form "identity + nuclear" one has a well-defined notion of determinant. If $E$ is a Banach space and $u=\sum_{j} e_{j} \otimes e_{j}^{*} \in$ $E \otimes E^{*}$, then one defines the trace by

$$
\operatorname{Tr}\left(v\left(\sum_{j} e_{j} \otimes e_{j}^{*}\right)\right)=\sum_{j} e_{j}^{*}\left(e_{j}\right)
$$

and for the determinant one has the formula

$$
\operatorname{det}(\operatorname{Id}+v(u))=1+\sum_{k=1}^{\operatorname{rank}(v(u))} \operatorname{Tr}\left(\bigwedge^{k} v(u)\right):=1+\sum_{k=1}^{\operatorname{rank}(v(u))} \alpha_{k}(u),
$$

where $\bigwedge^{k} v(u)$ is the $k$-th exterior power of $v(u)$ and each $\alpha_{k}$ is a $k$-homogeneous polynomial from $E \otimes E^{*}$ to $\mathbb{C}$. Now Grothendieck has shown in [Grot56] that all the $\alpha_{k}$ extend to $k$-homogeneous polynomials from $E \hat{\otimes} E^{*}$ to $\mathbb{C}$ and that $\sum_{k} \alpha_{k}(u)$ converges absolutely.

Recall that the map $v: E \hat{\otimes} E^{*} \mapsto L(E, E)$ is injective if and only if the space $E$ has the approximation property, see [Grot55]. Since this is the case for $E=H^{*}$, one can define for $v(u) \in$ $v\left(H^{*} \hat{\otimes}\left(H^{*}\right)^{*}\right)$, the analytic function

$$
\operatorname{det}(\operatorname{Id}+v(u))=1+\sum_{k=1}^{\infty} \alpha_{k}(u)
$$


It has the usual properties for a determinant: for two nuclear operators $u_{1}$ and $u_{2}$ on $H^{*}$

$$
\begin{array}{r}
\operatorname{det}\left(\left(\operatorname{Id}+u_{1}\right)\left(\operatorname{Id}+u_{2}\right)\right)=\operatorname{det}\left(\operatorname{Id}+u_{1}\right) \operatorname{det}\left(\operatorname{Id}+u_{2}\right) \\
, \operatorname{Id}+u_{1} \text { is invertible } \Leftrightarrow \operatorname{det}\left(\operatorname{Id}+u_{1}\right) \neq 0 .
\end{array}
$$

Now one can define holomorphic line bundles Det and $\operatorname{Det}^{*}$ over $\operatorname{Gr}_{0}(H)$, by dividing out on the product space $\mathcal{P} \times \mathbb{C}$ respectively the following two actions of $\mathcal{T}^{*}$,

$$
\begin{aligned}
(w, \lambda) & \mapsto\left(w \circ t^{-1}, \lambda \operatorname{det}(t)^{-1}\right), \\
(w, \lambda) & \mapsto\left(w \circ t^{-1}, \lambda \operatorname{det}(t)\right) .
\end{aligned}
$$

The class of $(w, \lambda)$ in Det is denoted by $[w, \lambda]_{1}$ and that in Det* by $[w, \lambda]_{2}$. As in the finite dimensional case, the line bundle Det* has nontrivial sections, e.g. $\sigma: \mathrm{Gr}_{0}(H) \mapsto$ Det* given by $^{*}$

$$
\sigma(\text { Image of } w)=\left[w, \operatorname{det}\left(w^{*}\right)\right]_{2} .
$$

The connected component $G l_{\mathfrak{n}}^{(0)}(H)$ of $G l_{\mathfrak{n}}(H)$ acts transitively on $\mathrm{Gr}_{0}(H)$ and to lift this action to the line bundles Det and Det* one has to pass to an extension $\mathscr{E}$ of $G l_{\mathfrak{n}}^{(0)}(H)$. It is defined by

$$
\mathcal{E}=\left\{(g, q)=\left(\left(\begin{array}{ll}
a & b \\
c & d
\end{array}\right), q\right) \mid \begin{array}{c}
\text { with } g \in G l_{\mathfrak{n}}^{(0)}(H), q \in G l\left(H^{*}\right) \\
\text { and } a q^{-1}-\text { Id nuclear }
\end{array}\right\} .
$$

The group $\&$ acts on Det and Det* by

$$
(g, q) \cdot[w, \lambda]_{i}=\left[g \circ w \circ q^{-1}, \lambda\right]_{i} .
$$

This induces an action of $\mathcal{E}$ on the holomorphic sections of Det*, in particular for $\sigma$ we get

$$
(g, q) . \sigma(\operatorname{Im}(w))=\left[w, \operatorname{det}\left(\left(g^{-1} \circ w \circ q\right)^{*}\right)\right]_{2} .
$$

Hence, if we define the function $\tau_{w}$ on $\&$ by

$$
\tau_{w}((g, q))=\operatorname{det}\left(\left(g^{-1} \circ w \circ q\right)^{*}\right)=\operatorname{det}\left(\left(a w^{*} q+b w^{+} q\right)^{*}\right), \text { for } g^{-1}=\left(\begin{array}{ll}
a & b \\
c & d
\end{array}\right),
$$

then this function measures the failure of the $\mathcal{E}$-equivariance of the section $\sigma$. Note that choosing another embedding from $\mathcal{P}^{*}$ with image $W$ only results in multiplying this function with a non zero constant.

The next step will be the construction of the wave functions of type $(i, \sigma(\underline{m}))$ of the multipoint $K P$-hierarchy.

\section{The construction of the multipoint wavefunctions}

For any plane $W$ belonging to $\operatorname{Gr}_{0}(H)$ one considers in the group $\Sigma^{(0)}$ the subset

$$
\Sigma_{W}^{(0)}=\left\{\begin{array}{l|l}
\sigma(\underline{m}) \mid \begin{array}{l}
\sigma(\underline{m}) \in \Sigma^{(0)}, \text { there is a } \gamma \in \tilde{\Gamma}(M) \text { such that } \\
p^{*}: \gamma^{-1} \sigma(\underline{m})^{-1} W \rightarrow H^{*} \text { is a bijection }
\end{array}
\end{array}\right\}
$$

Note that each $\sigma(\underline{m})$ belongs to $\Sigma_{W}^{(0)}$ for each $W$ in the inverse image under $\sigma(\underline{m})$ of the big cell. Since the translates of the big cell under the group $\Sigma^{(0)}$ cover $\operatorname{Gr}_{0}(H)$, one knows that the set $\Sigma_{W}^{(0)}$ is non-empty. 
Starting with a $\sigma(\underline{m})$ in $\Sigma_{W}^{(0)}$ one considers for a sufficiently large $M$ the group of continuous flows $\tilde{\Gamma}(M)$ and in it the open subset $\Gamma(\sigma(\underline{m}), W)$ given by

$$
\Gamma(\sigma(\underline{m}), W)=\left\{\gamma \mid \gamma \in \tilde{\Gamma}(M), \gamma^{-1} \sigma(\underline{m})^{-1} W \text { is transverse to } H^{*}\right\} .
$$

Let $\mathcal{R}$ be the ring of analytic functions on $\Gamma(\sigma(\underline{m}), W)$. Since for each $\gamma$ in $\Gamma(\sigma(\underline{m}), W)$, the plane $\gamma^{-1} \sigma(\underline{m})^{-1} W$ is transverse to $H^{*}$, this enables you to define for each $k, n \leq k \leq 1$,

$$
\hat{\psi}_{W, k}^{(\sigma(m))}(\gamma)=\left(\left.p^{*}\right|_{\gamma^{-1} \sigma(\underline{m})^{-1} W}\right)^{-1}\left(\left(\left(z-a_{k}\right)^{-1}, \cdots,\left(z-a_{k}\right)^{-1}\right)\right) .
$$

By multiplying with $\gamma \sigma(\underline{m})$ one translates the element $\hat{\psi}_{W, k}^{(\sigma(m))}(\gamma)$ in $\gamma^{-1} \sigma(\underline{m})^{-1} W$ back to $W$ and thus we obtain a $\psi_{W, k}^{(\sigma(\underline{m}))}: \Gamma(\sigma(\underline{m}), W) \rightarrow W$ that can be written as

$$
\begin{aligned}
\psi_{W, k}^{(\sigma(\underline{m}))}(\gamma) & =\hat{\psi}_{W, \underline{k}}^{(\sigma(\underline{m}))}(\gamma) \sigma(\underline{m}) e^{\xi} \\
& =\left\{\left(\ldots, \frac{1}{\left(z-a_{k}\right)^{1}}+\sum_{r \geq 0} \alpha_{r \ell}^{(k, j)}(\sigma(\underline{m}), \gamma)\left(z-a_{j}\right)^{r}, \ldots\right)\right\} \sigma(\underline{m}) e^{\xi} .
\end{aligned}
$$

Hence each function $\psi_{W, k}^{(\sigma(m))} \in \mathcal{M}(\mathcal{R})$ is of type $(k, \sigma(\underline{m}))$ and they are the candidate wavefunctions. To loosen up the notations one leaves out in the sequel the $W$-resp. $\sigma(\underline{m})$-dependence of the $\left\{\psi_{W, k}^{(\sigma(m))}\right\}$ and writes $\hat{\psi}_{k}$ resp. $\psi_{k}$ instead of $\hat{\psi}_{W, k}^{(\sigma(m))}$ resp. $\psi_{W, k}^{(\sigma(m))}$. The $j$-th component of $\hat{\psi}_{k}$ resp. $\psi_{k}$ is denoted by $\hat{\psi}_{k j}$ resp. $\psi_{k j}$ and the last one is given by

$$
\psi_{k j}(\gamma)=\left\{\frac{1}{\left(z-a_{k}\right)^{1}}+\sum_{r \geq 0} \alpha_{r \ell}^{(i, j)}(\sigma(\underline{m}), \gamma)\left(z-a_{j}\right)^{r}\right\} \sigma(\underline{m}) e^{\xi} .
$$

In a number of steps it will be shown that the $\psi_{i}$ satisfy the equations (10) and one starts with the $t_{k}^{(i)}$-derivatives of $\psi_{i}$.

Proposition 2 For all $k \geq 1$ and all $i, 1 \leq i \leq n$, there is a unique differential operator $P_{k}^{(i)}$ of the form $P_{k}^{(i)}=\sum_{s=0}^{k} p_{k s}^{(i)} \partial_{i}^{s}$, with $p_{k k}^{(i)}=1$, such that

$$
\partial_{k}^{(i)}\left(\psi_{i}\right)=P_{k}^{(i)}\left(\psi_{i}\right)
$$

The coefficients of the differential operator $P_{k}^{(i)}$ are polynomial expressions in the coefficients $\left\{\alpha_{r 1}^{(i, i)} \mid\right.$ $0 \leq r \leq k-1\}$ and their $\partial_{i}$-derivatives.

Proof 6.1 For each $s$ in $\mathbb{N}$, one has

$$
\partial_{i}^{s}\left(\psi_{i}\right)=\sum_{r=0}^{s}\left(\begin{array}{c}
s \\
r
\end{array}\right) \partial_{i}^{s-r}\left(\hat{\psi}_{i}\right) \frac{1}{\left(z-a_{i}\right)^{r}} \sigma(\underline{m}) e^{\xi} .
$$

This formula implies that, for $j \neq i$, the $j$-th component of $\partial_{i}^{s}\left(\psi_{i}\right)$ contains only positive powers of $\left(z-a_{j}\right)$ and that the $i$-th component of $\partial_{i}^{s}\left(\psi_{i}\right)$ looks like

$$
\left\{\frac{r_{i}}{\left(z-a_{i}\right)^{s+1}}+\text { "higher order in } z-a_{i} "\right\} \sigma(\underline{m}) e^{\xi} .
$$

On the other hand one has

$$
\partial_{k}^{(i)}\left(\psi_{i}\right)=\left\{\partial_{k}^{(i)}\left(\hat{\psi}_{i}\right)+\hat{\psi}_{i}\left(z-a_{i}\right)^{-k}\right\} \sigma(\underline{m}) e^{\xi},
$$


and from both relations one sees that $\left\{\partial_{k}^{(i)}\left(\psi_{i}\right)-\partial_{i}^{k}\left(\psi_{i}\right)\right\} \sigma(\underline{m})^{-1} e^{-\xi}$ has no singularities around the points $\left\{a_{j}, j \neq i\right\}$ and has a lower order singularity than $k+1$ around $a_{i}$. Therefore, the $j$ th component of $\left\{\partial_{k}^{(i)}\left(\psi_{i}\right)-\partial_{i}^{k}\left(\psi_{i}\right)\right\} \sigma(\underline{m})^{-1} e^{-\xi}$ for $j \neq i$ belongs to $H_{j}^{+}$and the $i$-th component $\left\{\partial_{k}^{(i)}\left(\psi_{i}\right)-\partial_{i}^{k}\left(\psi_{i}\right)\right\}$ looks like

$$
\left\{c_{t}\left(z-a_{i}\right)^{t}+\text { "higher order in } z-a_{i} ”\right\} \sigma(\underline{m}) e^{\xi}
$$

with $t>-k-1$ and $c_{t}$ a polynomial expression in the $\alpha_{r 1}^{(i, i)}, 0 \leq r \leq k-1$, and their $\partial_{i}$-derivatives. If $t<0$, then one continues to reduce the order of the singularity around $a_{i}$ and one considers

$$
\partial_{k}^{(i)}\left(\psi_{i}\right)-\partial_{i}^{k}\left(\psi_{i}\right)-c_{t} \partial_{i}^{-t-1}\left(\psi_{i}\right) .
$$

Continuing in this fashion, one ends up with a differential operator $P_{k}^{(i)}$ of the required form such that for all $\gamma \in \Gamma(\sigma(\underline{m}), W)$

$$
\partial_{k}^{(i)}\left(\psi_{i}\right)(\gamma)-P_{k}^{(i)}\left(\psi_{i}\right)(\gamma) \in H^{+} \sigma(\underline{m}) \gamma .
$$

These vectors, however, also belong to $W$ and, by construction, the space $W \cap H^{+} \sigma(\underline{m}) \gamma=\{0\}$. Hence one gets

$$
\partial_{k}^{(i)}\left(\psi_{i}\right)(\gamma)=P_{k}^{(i)}\left(\psi_{i}\right)(\gamma) .
$$

The differential operator $P_{k}^{(i)}$ is unique, because one easily shows, by comparing coefficients of the powerseries involved, that each differential operator $P=\sum \alpha_{s} \partial_{i}^{s}$ such that $P\left(\psi_{i}\right)=0$ has to be zero. This proves the propositon.

Next one considers the action of $\partial_{k}^{(j)}$, with $j \neq i$, and thus obtain the "mixed" equations.

Proposition 3 For all $k \geq 1$ and all $j \neq i$, there exists a unique differential operator $R_{k}^{(j, i)}$ of the form

$$
R_{k}^{(j, i)}=\sum_{s=0}^{k-1} r_{s}^{(j, i)} \partial_{j}^{s}
$$

satisfying

$$
\partial_{k}^{(j)}\left(\psi_{i}\right)=\frac{1}{\left(a_{i}-a_{j}\right)^{k}} \psi_{i}+R_{k}^{(j, i)}\left(\psi_{j}\right) .
$$

The coefficients of the differential operator $R_{k}^{j, i}$ are polynomial expressions in the functions $\left\{\alpha_{r 1}^{(i, j)} \mid\right.$ $0 \leq r \leq k-1\}$, the $\left\{\alpha_{r 1}^{(j, j)} \mid 0 \leq r \leq k-1\right\}$ and their $\partial_{j}$-derivatives.

Proof 6.2 Consider again the singularities of $\left\{\partial_{k}^{j}\left(\psi_{i}\right)\right\} \sigma(\underline{m})^{-1} e^{-\xi}$ around the points $\left\{a_{1}, \ldots, a_{n}\right\}$. Since

$$
\partial_{k}^{(j)}\left(\psi_{i}\right)=\left\{\partial_{k}^{(j)}\left(\hat{\psi}_{i}\right)+\hat{\psi}_{i}\left(z-a_{j}\right)^{-k}\right\} \sigma(\underline{m}) e^{\xi},
$$

there is a $k$-th order singularity around $a_{j}$ and a first order around $a_{i}$. The first can be eliminated as in the proof of proposition 2 with a suitable $(k-1)$-th order differential operator $R_{k}^{j, i}=$ $\sum_{0 \leq s \leq k-1} r_{s}^{(j, i)} \partial_{j}^{s}$. The second with a proper multiple of $\psi_{i}$. Then one has again for all $\gamma \in$ $\Gamma(\sigma(\underline{m}), W)$

$$
\partial_{k}^{(j)}\left(\psi_{i}\right)(\gamma)-\frac{1}{\left(a_{i}-a_{j}\right)^{k}} \psi_{i}(\gamma)-R_{k}^{(j, i)}\left(\psi_{j}\right)(\gamma) \in W \cap H^{+} \sigma(\underline{m}) e^{\xi}=\{0\} .
$$

The first term in the right hand side of the expression for $\partial_{k}^{(j)}\left(\psi_{i}\right)$ is fixed and since the action of the differential operators in $\partial_{j}$ on $\psi_{j}$ has no torsion, this equation also implies the uniqueness of $R_{k}^{(j, i)}$. 
The lowest order operator $R_{1}^{(j, i)}$ has a simple form. The equation from proposition 3 becomes then

$$
\begin{aligned}
\partial_{j}\left(\psi_{i}\right) & =\frac{1}{\left(a_{i}-a_{j}\right)} \psi_{i}+\left\{\alpha_{01}^{(i, j)}-\frac{1}{a_{i}-a_{j}}\right\} \psi_{j} \\
& :=\frac{1}{\left(a_{i}-a_{j}\right)} \psi_{i}+c_{W}^{(i, j)} \psi_{j}
\end{aligned}
$$

Note that for all $i \neq j, c_{H^{*}}^{(i, j)}=\frac{-1}{a_{i}-a_{j}}$ is non-zero. This holds more generally

Lemma 6.1 For each $W$ in $\operatorname{Gr}_{0}(H)$ and all $i \neq j$, the function $c_{W}^{(i, j)}$ is not identical zero.

From the construction of the functions $\left\{\psi_{k}\right\}$, one deduces directly that it suffices to prove this for all $W$ in the big cell corresponding to $H^{*}$. Then one uses that $c_{H^{*}}^{(i, j)} \neq 0$, the actual form of the flows in $\tilde{\Gamma}(M)$ and the analyticity of $c_{W}^{(i, j)}(\gamma)$ in $\gamma$ to prove the lemma.

To derive a relation between the candidate wavefunctions one restricts them now to the dense open part $\tilde{\Gamma}(\sigma(\underline{m}), W)$ of $\Gamma(\sigma(\underline{m}), W)$, given by

$$
\tilde{\Gamma}(\sigma(\underline{m}), W)=\left\{\gamma \mid \gamma \in \Gamma(\sigma(\underline{m}), W), c_{W}^{(i, j)}(\gamma) \neq 0 \text { for all } i \neq j\right\} .
$$

Clearly this implies that the ring $\mathcal{R}$ has to be adapted to that of the analytic functions on $\tilde{\Gamma}(\sigma(\underline{m}), W)$ and one introduces the differential operators $T_{j i}$ by

$$
T_{j i}=\frac{1}{c_{W}^{(i, j)}}\left(\partial_{j}-\frac{1}{a_{i}-a_{j}}\right) .
$$

The interrelations between the wavefunctions $\left\{\psi_{1}, \ldots, \psi_{n}\right\}$ are resumed in the following proposition

Proposition 4 For $i \neq j$ and $\gamma \in \tilde{\Gamma}(\sigma(\underline{m}), W)$ one has

$$
T_{j i}\left(\psi_{i}\right)(\gamma)=\psi_{j}(\gamma) .
$$

With the help of the operators $\left\{T_{j i}\right\}$ one can put the equations from the propositions 2 and 3 in the same form: for each $k \geq 1$ and all $i$ and $j$, there is differential operator $B_{k}^{(j, i)}$ of order $k$ in $\partial_{j}$ such that

$$
\partial_{k}^{(j)}\left(\psi_{i}\right)=B_{k}^{(j, i)}\left(\psi_{i}\right) .
$$

This shows that each $\psi_{i}$ is a wavefunction of type $(i, \sigma(\underline{m}))$ and it suffices to have one of them, since the others can be derived by differentiating this one.

Until now one has restricted oneself to the equations coming from the continuous flows from $\tilde{\Gamma}(M)$. One concludes with taking the action of $\Sigma^{(0)}$ into account. Assume that $\sigma_{1}$ and $\sigma_{1} \sigma_{2}$ belong to $\Sigma_{W}^{(0)}$. One has then wavefunctions $\psi_{W, k}^{\left(\sigma_{1}\right)}$ and $\psi_{W, k}^{\left(\sigma_{1} \sigma_{2}\right)}$ and as in [HP94] these solutions corresponding to different sites in the lattice $\Sigma^{(0)}$ are connected by a differential operator

Proposition 5 There is a differential operator $R_{\sigma_{1}}^{\sigma_{1} \sigma_{2}} \in \mathcal{R}\left[\partial_{j}, 1 \leq j \leq n\right]$ with $\mathcal{R}$ the ring of analytic functions on $\tilde{\Gamma}\left(\sigma_{1}, W\right) \cap \tilde{\Gamma}\left(\sigma_{1} \sigma_{2}, W\right)$ such that

$$
\psi_{W, k}^{\left(\sigma_{1} \sigma_{2}\right)}=R_{\sigma_{1}}^{\sigma_{1} \sigma_{2}}\left(\psi_{W, k}^{\left(\sigma_{1}\right)}\right)
$$

Proof 6.3 Since $\sigma_{2}$ belongs to $\Sigma^{(0)}$, it is the product of the elements

$$
\sigma_{i j}:=\frac{\left(z-a_{i}\right)}{\left(z-a_{j}\right)}=1-\frac{a_{i}-a_{j}}{z-a_{j}} .
$$


Hence $\sigma_{2}$ is a polynomial in the $\left\{\frac{1}{z-a_{j}} \mid 1 \leq j \leq n\right\}$ and let its highest order term be given by

$$
c \prod_{j=1}^{n}\left(\frac{1}{z-a_{j}}\right)^{s_{j}}, \text { with } s_{j} \geq 0 \text { and } c \in \mathbb{C}, c \neq 0 \text {. }
$$

Consider then the function

$$
\left(\psi_{W, k}^{\left(\sigma_{1} \sigma_{2}\right)}-c \prod_{j=1}^{n} \partial_{j}^{s_{j}}\left(\psi_{W, k}^{\left(\sigma_{1}\right)}\right)\right) \sigma_{1}^{-1} e^{-\xi}
$$

It has on each component singularities in the points $\left\{a_{1}, \ldots, a_{n}\right\}$ of order less then $\sum_{j} s_{j}$. Hence by subtracting from $\psi_{W, k}^{\left(\sigma_{1} \sigma_{2}\right)}$ a finite number of terms of the form $\prod_{j=1}^{n} \partial_{j}^{n_{j}}\left(\psi_{W, k}^{\left(\sigma_{1}\right)}\right)$ one can remove by a step by step procedure the singularities in all components and finally one ends up with a differential operator $R_{\sigma_{1}}^{\sigma_{1} \sigma_{2}} \in \mathcal{R}\left[\partial_{j}, 1 \leq j \leq n\right]$ such that

$$
\psi_{W, k}^{\left(\sigma_{1} \sigma_{2}\right)}-R_{\sigma_{1}}^{\sigma_{1} \sigma_{2}}\left(\psi_{W, k}^{\left(\sigma_{1}\right)}\right) \in W \cap H^{+} \sigma_{1} e^{\xi}=\{0\} .
$$

This proves the claim in the proposition.

Remark 2 Note in particular that proposition 4 implies that each wavefunction $\psi_{i}$ is annihilated by each of the second order differential operators $T_{i j} T_{j i}-1$, with $j \neq i$. Hence one can not, contrary to the case of the KP-hierarchy, scratch the wavefunction in the equations (11).

Remark 3 We have associated to each plane $W$ in $\operatorname{Gr}_{0}(H)$ a set of wavefunctions $\left\{\psi_{W, k}^{(\sigma(m))} \mid 1 \leq\right.$ $i \leq n\}$ of which each member determines the others. There holds even something stronger: the whole space $W$ can be recovered from any of these wavefunctions by taking arbitrary derivatives w.r.t. the variables $\left\{t_{1}^{(i)}\right\}$.

Remark 4 Since the computations in [Hel03] for the relation between the wavefunction and the $\tau$-function in the $L^{2}$-case are based on the formula (24), they carry over to the present more general setting with $\tau_{w}$ defined as in section 5.

\section{References}

[DJKM83] E. Date, M. Jimbo, M.Kashiwara, T. Miwa, Transformation groups for soliton equations, in: Nonlinear integrable systems - Classical theory and quantum theory eds. M. Jimbo, and T. Miwa, World Scientific, 39-120, 1983.

[Dic94] L.A. Dickey, Why the general Zakharov-Shabat equations form a hierarchy?, Commun. Math. Phys., 163:509-521, 1994.

[dRoe78] J.W. de Roever, Complex Fouriertransformation and analytic functionals with unbounded carriers, Mathematical Centre Tracts 89, 1978.

[Dorf89] Dorfmeister, Neher and J.Szmigielski, Automorphisms of Banach manifolds associated with the KP-equation

[Grot55] A.Grothendieck, Produits tensoriels topologiques et espaces nucléaires, Mem.Amer.Math.Soc.16(1955).

[Grot56] A.Grothendieck, La Théorie de Fredholm, Bull.Soc.math. de France (84) (1956), p.319384. 
[Hel03] G.F. Helminck, $\tau$-functions for a two-point version of the KP-hierarchy, to appear in Indag. Math.

[HP94] G.F. Helminck and G.F. Post, The geometry of differential difference equations, Indag. Mathemat., N.S., 5(4) (1994), 411-438.

[Kac88] V.G. Kac., Infinite dimensional Lie algebras, volume 44 of Progress in mathematics, Birkhäuser, third edition, 1988.

[SS83] Mikio Sato and Yasuko Sato, Soliton equations as dynamical systems on infinitedimensional Grassmann manifold, Nonlinear partial differential equations in applied science (Tokyo, 1982), North-Holland Math. Stud., vol. 81, North-Holland, Amsterdam, 1983, pp. 259-271.

[SW85] G. Segal and G. Wilson, Loop groups and equations of KdV type, Publ. Math. IHES 63 (1985), 1-64. 